\title{
Oportunidades y desafíos de las Fintech en Perú
}

DOI: $10.46932 /$ sfjdv2n1-014

Received in: November 1st, 2020

Accepted in: December 30th, 2020

\section{Carlos Rios-Campos}

Docente investigador RENACYT. Doctor en Gestión Universitaria. Universidad Politécnica Amazónica.

Chiclayo, Perú.

E-mail: crios@upa.edu.pe

\section{Freddy Camacho Delgado}

Docente Principal. Doctor en Economía. Universidad Nacional Intercultural Fabiola Salazar Leguía de Bagua. Bagua Grande, Perú.

E-mail: fcamacho@ unibagua.edu.pe

\section{Henrry Agreda Cerna}

Docente asociado. Maestro en Administración de la Educación. Universidad Nacional José María Arguedas. Andahuaylas, Perú.

E-mail: hwagredacerna@gmail.com

\section{Italo Maldonado Ramirez}

Decano de la Facultad de Ingeniería de Sistemas y Mecánica Eléctrica. Doctor en Administración de la Educación. Universidad Nacional Toribio Rodríguez de Mendoza de Amazonas. Bagua, Perú. E-mail: italo.maldonado@untrm.edu.pe

\section{Roberto Santa Cruz Acosta}

Director Departamento Académico de Ingeniería. Maestro en Ingeniería de Sistemas. Universidad Nacional Toribio Rodríguez de Mendoza de Amazonas. Bagua, Perú.

E-mail: roberto.santacruz@untrm.edu.pe

\section{RESUMEN}

Resulta necesario conocer el estado de arte de las fintech en Perú, considerando que a nivel mundial las startups financieras tienen un crecimiento importante y un impacto social y económico. Se planteó el objetivo general, analizar las oportunidades y desafíos de las fintech en Perú. Metodología, la investigación presenta un diseño cualitativo-interpretativo, de tipo documental, se han seleccionado 27 documentos, realizados en el periodo 2015 - 2020; incluyendo: artículos científicos, artículos de revisión e información de sitios web de organizaciones reconocidas. Las palabras claves utilizadas en las búsquedas fueron: fintech e inclusión financiera. Resultados, en el Perú las principales fintech limeñas, están en un proceso de posicionamiento de mercado. Además, se requiere el decidido apoyo a los emprendedores de provincias, con estímulos financieros, capacitaciones y mentorías. Conclusiones, con respecto al objetivo general, analizar las oportunidades de las fintech en Perú, se observó el apoyo inicial por parte del Estado Peruano, mediante el Decreto de Urgencia $N^{\circ}$ 013-2020, Decreto de Urgencia que promueve el financiamiento de la MIPYME, Emprendimientos y Startups; el mercado de las fintech está creciendo en provincias; la Asociación Fintech del Perú, está ayudando a construir un ecosistema fintech sólido. Al analizar los desafíos de las fintech en Perú, destacan la urgencia de reducir las transacciones que se hacen de manera física, aumentar la inclusión financiera y el escaso financiamiento.

Palabras clave: Inclusión financiera, fintech, startup, Perú. 


\section{INTRODUCCIÓN}

\subsection{INCLUSIÓN FINANCIERA}

La inclusión financiera significa, para personas físicas y empresas, tener acceso a productos financieros útiles y asequibles que satisfagan sus necesidades - transacciones, pagos, ahorros, crédito y seguro_- prestados de manera responsable y sostenible (Banco Mundial, 2020).

Una mayor cobertura de los servicios financieros ayuda a las personas de menores recursos a acceder a fuentes de financiación de su consumo, liberando ingresos para invertir en aspectos fundamentales de su proyecto de vida como su educación (Franco, 2017).

Además, 1.700 millones de adultos en todo el mundo no disponen de una cuenta bancaria, de los cuales 1.100 millones disponen, no obstante, de un teléfono móvil... En Kenia, la expansión del dinero móvil permitió sacar de la pobreza un dos por ciento de los hogares del país (1,90 USD al día) (ITU, 2020).

Un ejemplo de las oportunidades y desafíos analizados por el informe son las nuevas tecnologías y la tecnología financiera o fintech (innovación digital habilitada en el sector financiero). Más de 500 millones de personas han obteniendo acceso a servicios financieros en los últimos años, por lo que el atractivo de la fintech es evidente (ONU, 2019).

\subsection{FINTECH}

Los nuevos operadores, como las firmas de fintech, apuntan hacia aspectos rentables del negocio bancario, el cual, a su vez, se encuentra en un punto de inflexión con una creciente amenaza de interrupción. Es decir, el acecho de los competidores no tradicionales ha ido ganando fuerza en la industria financiera, ya que estas empresas están dirigidas a diferentes servicios bancarios, tales como pagos, administración de finanzas personales, préstamos, inversiones y actividades de banca central (Ramos \& Borrás, 2017).

La banca tradicional hoy en día está amenazada ante la llegada de las empresas fintech, y aunque algunas de ellas se han asociado o han sido adquiridas por los bancos, se estima que desplazarán de manera significativa a la banca en varios segmentos de su negocio, sobre todo en poblaciones más jóvenes (Avendaño, 2018).

Los participantes destacaron la importancia de un marco regulatorio que favorezca la entrada de nuevos actores; el papel de Fintech y de la regulación para facilitar los requerimientos de "conocer a su cliente"; fortalecimiento de la interoperabilidad; y una gestión de riesgos apropiada (FMI, 2019).

China ha establecido un centro nacional de certificación de tecnología financiera (fintech) en la municipalidad de Chongqing, dijo el Banco Popular de China (BPCh). El establecimiento del centro en 
Chongqing marcó un paso significativo en la implementación del Plan de Desarrollo de Tecnología Financiera (2019-2021) y es una medida activa para mejorar el marco regulador para la fintech, dijo Fan Yifei, vicegobernador del BPCh. Se espera que se convierta en una autoridad de certificación de primera clase y profesional, sirva como un "guardián" de la integridad, la innovación, la seguridad y el cumplimiento en el desarrollo de fintech, y se esfuerce por convertirse en la columna vertebral del sistema de gobernanza conjunta para fintech en China, fortaleciendo así el desarrollo de calidad del sector financiero, dijo (Xinhuanet, 2020).

En Europa, de las 481 rondas de financiación llevadas a cabo en 2019, nueve fintech acapararon el $42 \%$ de la inversión. Nueve de estas 10 firmas son británicas o alemanas. No hay ninguna española en esta lista. La startup de Europa que obtuvo más financiación es la germana Wirecard, plataforma de pagos, con 900 millones de euros de recaudación, y cuyo principal inversor es Softbank (Gonzalo, 2020).

El crecimiento del sector Fintech en Canadá cuenta con varias barreras de entrada entre las que destacan la compleja estructura regulatoria del país al respecto, los altos costes requeridos para el cumplimiento de las normativas existentes y el bajo nivel de acceso a financiación, de acuerdo con un estudio del Competition Bureau in Canada (ICEX, 2020).

América Latina no se ha quedado al margen de esta evolución, produciéndose un marcado crecimiento en los emprendimientos fintech, mientras que la regulación en la mayoría de los países es aún incipiente. En 2019 el sector fintech en Uruguay estaba compuesto por 63 empresas, operando en diversos segmentos (Lavalleja, 2020). Además, México es uno de los países con mayor ecosistema fintech en América Latina, pues gran variedad de plataformas en distintos sectores tecnológico-financieros han prosperado en el país, en especial empresas de crowdfunding (García, 2019).

\section{METODOLOGÍA}

La investigación presenta un diseño cualitativo-interpretativo, de tipo documental, el cual precisó el procedimiento de selección y el registro de los datos (Barrero y Rosero, 2018).

En la presente investigación se han seleccionado 27 documentos, realizados en el periodo 2015 2020; incluyendo: artículos científicos, artículos de revisión e información de sitios web de organizaciones reconocidas. Las palabras claves utilizadas en las búsquedas fueron: fintech e inclusión financiera. Para la selección de los documentos se usaron como criterios: el año de la publicación, la pertenencia con la investigación y ser una fuente confiable. Después de la lectura de cada documento, se procedió a ingresar los datos a la matriz bibliográfica, que sirve para catalogar los documentos de acuerdo con categorías, que se presentan en la figura 1. 
Figura 1. Matriz bibliográfica

\begin{tabular}{|l|l|l|l|}
\hline Nombre & Tipo & Objetivos & Conclusiones \\
\hline & & & \\
\hline & & & \\
\hline
\end{tabular}

Fuente: Adaptado de Barrero y Rosero (2018).

\section{RESULTADOS}

\subsection{DESAFÍOS DE LAS FINTECH EN PERÚ}

Fernando Eguiluz, CEO de BBVA en Perú, en una entrevista concedida para la agencia de noticias EFE, afirmó que “en Perú más del 80\% de las transacciones se hacen de manera física, así que estamos también trabajando mucho a nivel de comercio para que se pueda recibir de manera mucho más fácil el tema de los pagos y terminar procesos end to end de manera digital” (BBVA, 2020).

Según la encuesta realizada por el Global Findex en 2017, hay cuatro razones principales, que hasta ese entonces derivaban en que solo el $29 \%$ de la población adulta tuviera una cuenta financiera: (i) falta de dinero, (ii) altos costos de mantener una cuenta, (iii) desconfianza en el sistema financiero, y (iv) la distancia al punto de acceso más cercano al sistema financiero (Carballo \& Dalle-Nogare, 2019).

Este estudio en Perú muestra que las fintech analizadas innovan por la alta competitividad en el sector, la necesidad de crecer y, principalmente, porque su propio modelo de negocio así lo exige. Sin embargo, existen barreras que dificultan sus innovaciones como la falta de prioridad de parte de sus aliados, el acceso a financiamiento y la demora de la regulación respecto a nuevas tecnologías como la identidad digital (Palomino, Velásquez, Marcos \& Seclen, 2019).

\subsection{OPORTUNIDADES DE LAS FINTECH EN PERÚ}

La Superintendencia de Banca, Seguros y AFP mencionó en el Foro que viene evaluando una propuesta regulatoria integral para incorporar a las fintech en el marco normativo sin limitar su desarrollo y crecimiento (Silva, 2017).

Que, conforme al literal g) del artículo 2 del Decreto de Urgencia $\mathrm{N}^{\circ}$ 013-2020, Decreto de Urgencia que promueve el financiamiento de la MIPYME, Emprendimientos y Startups, dicha norma tiene como finalidad impulsar el desarrollo productivo y empresarial a través de instrumentos de servicios no financieros, tales como iniciativas Clúster a nivel nacional, fortalecimiento e incentivo de los procesos de internacionalización de la MIPYME, así como de empresas exportadoras a que se refiere el Decreto Legislativo $\mathrm{N}^{\circ} 1403$, Decreto Legislativo que impulsa el fortalecimiento de las empresas exportadoras en el fondo MIPYME, la promoción del acceso al financiamiento de emprendimientos dinámicos y de alto impacto, y la ampliación de los servicios tecnológicos que brinda el Estado (Decreto de Urgencia 013, 2020). 
Este paquete de beneficios que incorpora el D.U. 013-2020 es un paso necesario para el desarrollo de la industria Venture capital en el Perú y se espera que abran las puertas al desarrollo de modelos de negocio innovadores y más competitivos (Vodanovic, 2020).

En nuestro país, este fenómeno no ha sido ajeno. Muchas actividades que normalmente los peruanos realizábamos de manera presencial han tomado una forma virtual por completo; dos buenos ejemplos son: (i) cambio de divisas y (ii) pagos digitales (Vodanovic y Morán, 2020).

Asimismo, si bien entre el $88 \%$ y $90 \%$ de las fintech se concentran en Lima, el mercado está creciendo en provincias. En ese sentido, la ejecutiva espera una mayor descentralización de este segmento en las regiones, y que en ciudades como Arequipa, Cusco, Tacna y el norte del país se está viendo emprendimientos financieros basados en tecnología. "Arequipa, por ejemplo, se ha convertido en un hub para la innovación en general”, destaca María Laura Cuya (López, 2020).

La Asociación Fintech del Perú (2018), fue fundada en setiembre del 2017, tiene más de 73 empresas asociadas y afiliadas. Tiene como propósito, construir un ecosistema Fintech sólido, inclusivo, innovador de productos y servicios financieros formados en principios confiables y seguros, con las mejores prácticas de la industria fintech, en colaboración con stakeholders como comunidad, el gobierno, el sector financiero. Buscamos generar un ecosistema robusto y empoderando, a través de principios como la Transparencia y Seguridad, Innovación Educación e Inclusión, Colaboración y Comunidad.

La especialista en emprendimientos de Innóvate Perú, Evelyn Gómez, presentó la siguiente figura 1.

Figura 1: 130 Fintech identificadas en Perú.

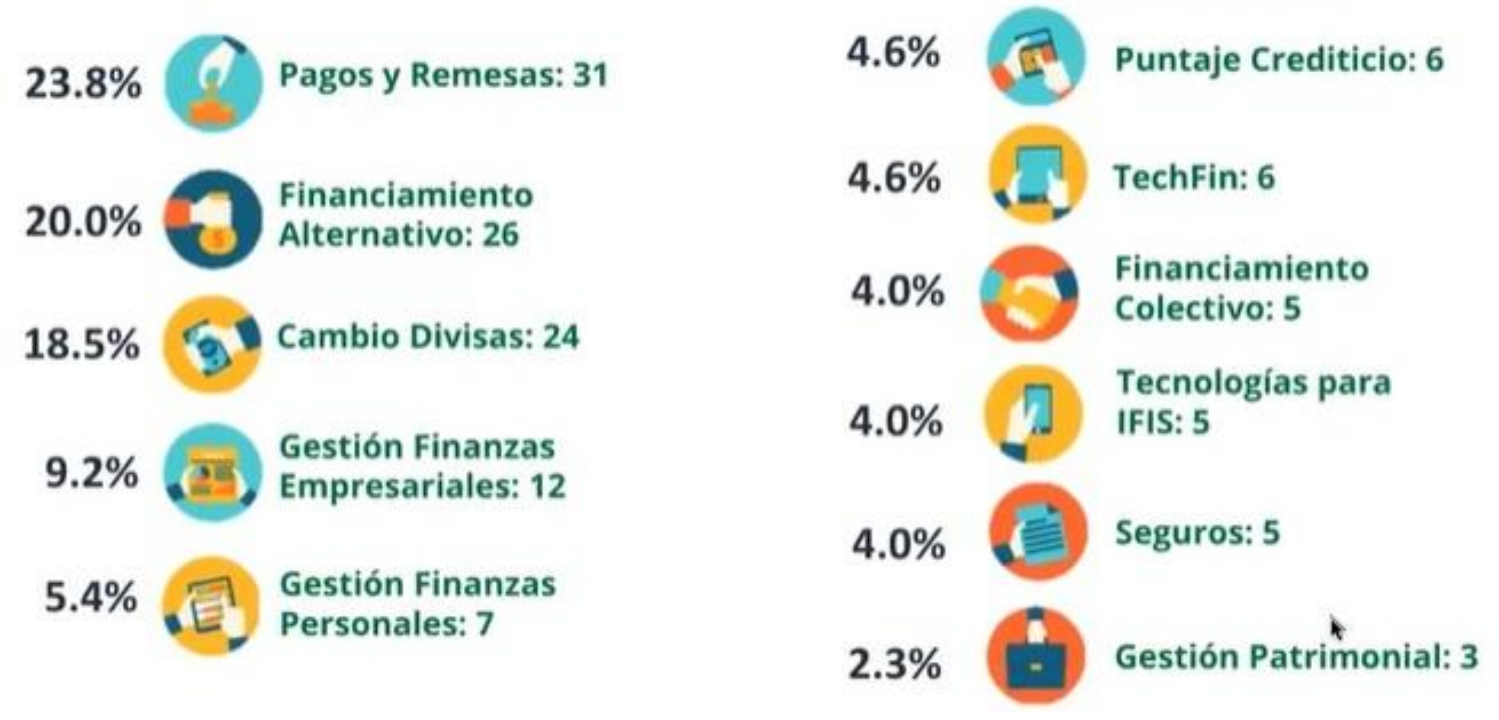

Fuente: Asociación Fintech del Perú / Perú Incuba (Infomercado, 2019). 
Figura 2: + 50 inician actividades a partir del 2016

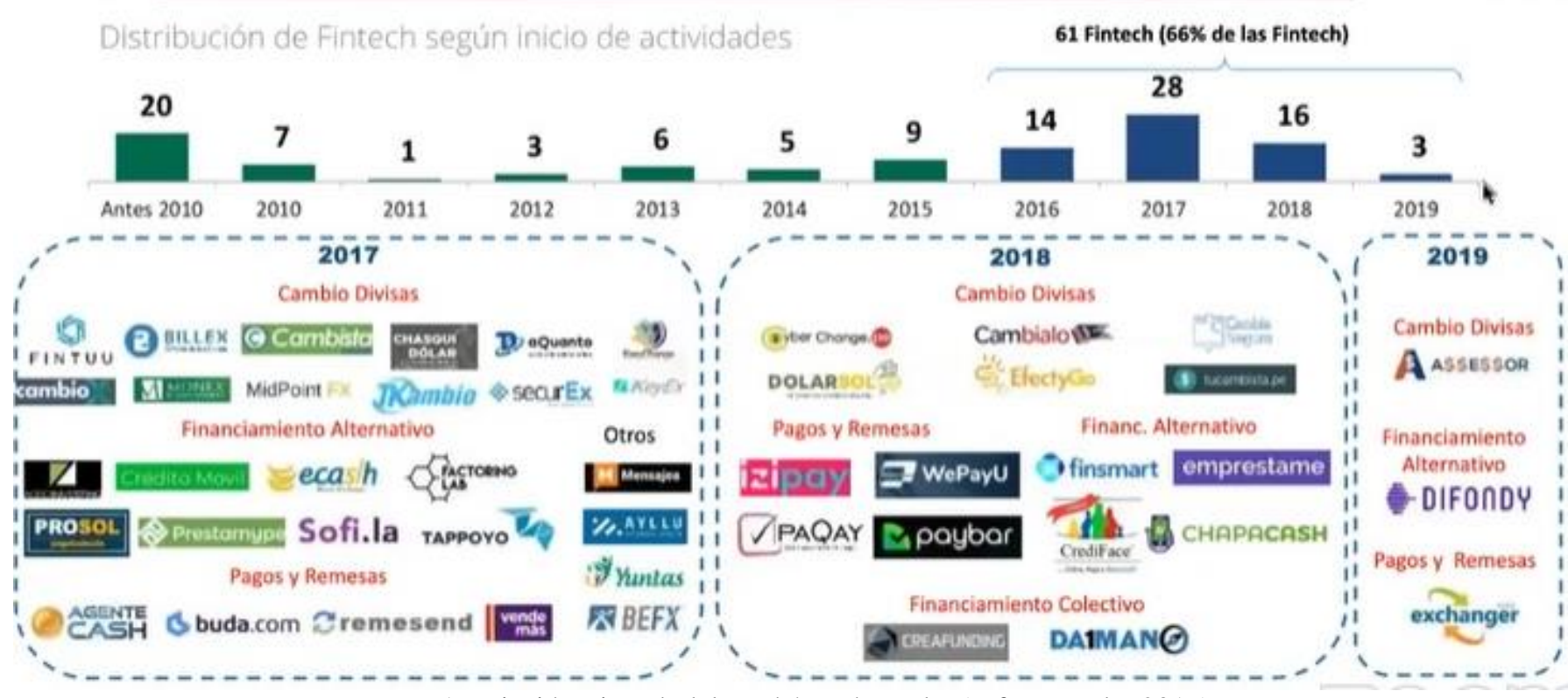

Fuente: Asociación Fintech del Perú / Perú Incuba (Infomercado, 2019).

El 61\% de las 151 fintechs que hay el Perú se concentra en los rubros de pagos, préstamos y casas de cambio, detectó el estudio Panorama del Ecosistema Fintech 2020, elaborado por Equifax y el Centro de Emprendimiento e Innovación de la Universidad del Pacífico (Emprende UP). Por otro lado, si se analiza por nivel de endeudamiento, el rubro "techfin" (métodos de pago, como Yape, Plin, Lukita, etc.) acumula S/22,3 millones en créditos a junio de 2020, el triple que el segundo rubro "Puntaje crediticio" (S/7,7 millones), seguido por "Préstamos" (S/6,3 millones), "Casas de cambio" (S/5,2 millones) y "Pagos" (S/4 millones) (El Comercio, 2020).

Salkantay Partners, una entidad que gestiona inversiones en mercados emergentes, lanzará un fondo de venture capital para apoyar a empresas innovadoras nacionales con algún componente tecnológico y alto potencial de expansión en la región, mediante la compra de acciones... Los sectores en los que tendrán presencia son e-commerce, logística de última milla, healthtech, fintech y soluciones digitales para otras empresas (Innovateperu, 2019).

En el Perú las principales fintech limeñas, están en un proceso de posicionamiento de mercado. Además, se requiere el decidido apoyo a los emprendedores de provincias, con estímulos financieros, capacitaciones y mentorías.

\section{CONCLUSIONES}

Con respecto al objetivo general, analizar las oportunidades de las fintech en Perú, se observó el apoyo inicial por parte del Estado Peruano, mediante el Decreto de Urgencia N ${ }^{\circ} 013-2020$, Decreto de Urgencia que promueve el financiamiento de la MIPYME, Emprendimientos y Startups; el mercado de 
las fintech está creciendo en provincias; la Asociación Fintech del Perú, está ayudando a construir un ecosistema Fintech sólido.

Al analizar los desafíos de las fintech en Perú, destacan la urgencia de reducir las transacciones que se hacen de manera física, aumentar la inclusión financiera y el escaso financiamiento. 


\section{REFERENCIAS BIBLIOGRÁFICAS}

Asociación Fintech del Perú (2018). Acerca de la comunidad de fintech peruanas Asociación Fintech del Perú. Recuperado de https://www.fintechperu.com/

Avendaño Carbellido, Octavio. (2018). Los retos de la banca digital en México. Revista IUS, 12(41), 87108. Recuperado de http://www.scielo.org.mx/scielo.php?script=sci_arttext\&pid=S1870$21472018000100087 \& \operatorname{lng}=$ es\&tlng=es.

Banco Mundial (2020). La inclusión financiera es un factor clave para reducir la pobreza e impulsar la prosperidad. Recuperado de https://www.bancomundial.org/es/topic/financialinclusion/overview

Barrero, A. y Rosero, A. (2018). Estado del Arte sobre Concepciones de la Diversidad en el Contexto Escolar Infantil. Revista Latinoamericana de Educación Inclusiva, 2018, 12(1), 39-55 https://doi.org/10.4067/S0718-73782018000100004

BBVA (2020). La banca digital y la inclusión financiera son los desafíos en medio de la COVID-19. Recuperado de https://www.bbva.com/es/la-banca-digital-y-la-inclusion-financiera-son-los-desafios-enmedio-de-la-covid-19/amp/

BID (2020). BID Lab impulsará la inclusión financiera digital de pequeños productores rurales de Bolivia. Recuperado de https://bidlab.org/es/noticias/1652/bid-lab-impulsara-la-inclusion-financieradigital-de-pequenos-productores-rurales-de

Carballo, I. E., \& Dalle-Nogare, F. (2019). Fintech e inclusión financiera: los casos de México, Chile y Perú. Revista CEA, 5(10), 11-3. https://doi.org/10.22430/24223182.1441

Decreto de Urgencia 013 (2020). Presentación general de las medidas del Decreto de Urgencia $n .^{\circ} 013$ 2020. Recuperado de https://www.gob.pe/institucion/produce/informes-publicaciones/444890presentacion-general-de-las-medidas-del-decreto-de-urgencia-n-013-2020

El Comercio (2020). Financiamiento de sector fintech aumentó 39\%, pese a impacto del COVID-19. Recuperado de https://elcomercio.pe/economia/peru/financiamiento-de-sector-fintech-aumento-39-pesea-impacto-del-covid-19-nndc-noticia/?ref=ecr

FMI (2019). Conclusiones de la XVI Conferencia Regional sobre Centroamérica, Panamá y la República Dominicana. Recuperado de https://www.imf.org/es/News/Articles/2019/11/15/pr19419-conclusionsxvi-regional-conference-on-central-america-panama-and-the-dominican-republic

Franco, P. (2017). Tecnología, inclusión financiera y regulación: acercando el financiamiento a las personas. $21^{\circ}$ Conferencia anual de la Asociación Latinoamericana e Ibérica de Derecho y Economía $(A L A C D E)$ - Universidad del Pacífico - Perú. Recuperado de https://www.up.edu.pe/UP_Landing/alacde2017/papers/25-Tecnologia-inclusion-financieraregulacion.pdf

García Gallegos, Daniel. (2019). Crowfunding, transformación digital financiera y jurídica en México. Revista chilena de derecho y tecnología, 8(2), 139-155. https://dx.doi.org/10.5354/07192584.2019.52179 
Gonzalo, A. (2020). España se sitúa a la cola en inversión en 'fintech' de Europa con solo el 3\%. Recuperado de https://cincodias.elpais.com/cincodias/2020/01/24/companias/1579892539_469024.html ICEX (2020). Fintech en Canadá. Recuperado de https://www.cnbs.gob.hn/wpcontent/uploads/2019/11/Fintech-en-Canad\%C3\%A1.pdf

Infomercado (2019). Fintech Perú: ¿Cómo se puede enfrentar la precaria inclusión financiera?. Recuperado de https://infomercado.pe/fintech-peru-como-se-puede-enfrentar-la-precaria-inclusionfinanciera

Innovateperu (2019). Lanzan fondo de capital de riesgo para apoyar a emprendedores. Recuperado de https://www.innovateperu.gob.pe/noticias/noticias/item/1963-lanzan-fondo-de-capital-de-riesgo-paraapoyar-a-emprendedores

ITU (2020). Inclusión financiera digital. Inclusión financiera digital. Recuperado de https://www.itu.int/es/mediacentre/backgrounders/Pages/digital-financial-inclusion.aspx

Lavalleja, M. (2020). Panorama de las fintech: principales desafíos y oportunidades para el Uruguay. Recuperado de http://repositorio.cepal.org/bitstream/handle/11362/45727/1/S2000326_es.pdf

López (2020). La expansión de las fintech en el Perú. Recuperado de https://apps.camaralima.org.pe/repositorioaps/0/0/par/edicion914/revista_digital_914.pdf

Lozano, R. (2020). Auge de constitución en el país de empresas 'fintech'. Recuperado de https://forbes.co/2020/02/10/tecnologia/auge-de-constitucion-en-el-pais-de-empresas-fintech/

ONU (2019). Nuevo Informe de la ONU aboga por la revisión del Sistema Financiero Global. Recuperado de https://developmentfinance.un.org/nuevo-informe-de-la-onu-aboga-por-la-revisi\%C3\%B3n-delsistema-financiero-global

Palomino Barchi, G. J., Velásquez, V., Marcos Quispe, K. C., \& Seclen Luna, J. P. (2019). ¿Cómo innovan las fintech peruanas? Una aproximación a partir de un estudio de casos múltiple. 360: Revista De Ciencias De La Gestión, (4), 38-66. https://doi.org/10.18800/360gestion.201904.002

Ramos López, Elizabeth, \& Borrás Atiénzar, Francisco. (2017). Las relaciones crediticias entre las empresas y la banca comercial en Cuba. Cofin Habana, 11(2), 397-410. Recuperado de http://scielo.sld.cu/scielo.php?script=sci_arttext\&pid=S2073-60612017000200027\&lng=es\&tlng=es.

Silva, M. (2017). El acelerado crecimiento de las fintech y los desafios para su regulación. Recuperado de https://www.bcrp.gob.pe/docs/Publicaciones/Revista-Moneda/moneda-171/moneda-171-08.pdf

Vodanovic, L. (2020). Los beneficios del Decreto de Urgencia 013-2020 para el financiamiento de mipymes, startups y emprendimientos. Recuperado de https://www.ey.com/es_pe/law/beneficiosfinanciamiento-mipymes

Vodanovic, L. y Morán, A. (2020). FinTechs en el Perú: oportunidades emergentes durante el COVID19. Recuperado de https://www.ey.com/es_pe/banking-capital-markets-digital-innovation/fintechs-peruoportunidades-emergentes

Xinhuanet (2020). China establece centro nacional de certificación de tecnología financiera en Chongqing. Recuperado de http://spanish.xinhuanet.com/2020-10/19/c_139449803.htm 